\title{
Application of the solid-phase micro-extraction technique coupled to GC-MS in the control of the impact of the pest treatment products in the industrial unit on the industrialized food products Quality
}

\author{
Ahmed Lakhili ${ }^{1,2, *}$, Mohammed Fekhaoui ${ }^{3}$, Abdelkbir Bellaouchou ${ }^{2}$, Abdallah El Abidi ${ }^{2}$ and \\ Mohammed El idrissi ${ }^{4}$ \\ ${ }^{1}$ Mohammed V University, Rabat, Morocco \\ ${ }^{2}$ National Institute of Hygiene, Rabat, Morocco \\ ${ }^{3}$ Institut Scientifique, Rabat, Morocco \\ ${ }^{4}$ Chouaïb Doukkali University, El Jadida, Morocco
}

\begin{abstract}
In this investigation, solid phase extraction (SPME) is applied in order to determine the concentrations of insecticides and rat poisons detected in the samples taken on the industrial finished product. Gas chromatography, coupled with mass spectroscopy (GC-MS) is used as an analytical instrument for this purpose. In order to obtain an optimal extraction, different process parameters are examined and optimized. Relevant results are obtained using 30 minutes non-specific binding property time, with 30 minutes stirring mode with magnetic stirring and a temperature of $30{ }^{\circ} \mathrm{C}$. The efficiency of insecticides, poisons for rats, has been demonstrated, and pesticides are likely to be at a high concentration in finished industrial products.
\end{abstract}

Keywords: GC-MS; SPME; extraction; Performance; rat poisons; Insecticides.

\section{Introduction}

Pesticides (rat poisons and insecticides) are compounds characterized by their diversity and their different physicochemical properties ${ }^{1-2}$. Their adverse side effects have been quickly identified. It turns out that the toxicity related to their molecule structure is, in principle, not limited due to the associated species which should be evinced. It has been demonstrated that they are particularly toxic, not only to humans but also to the environment ${ }^{3-4}$. These molecules tend to accumulate in different biotic and abiotic matrices, including water, air, soil, aquatic organism, blood and food ${ }^{5-18}$. It is noted that pesticides are among the oldest organic synthetic chemicals used in agriculture activities all over the world, since 1940, due to their energetic efficiency against pests and diseases ${ }^{6-7}$. However, pesticides are very toxic and persistent in the environment, which tends to accumulate in living organisms. Due to their low degradation and high solubility in organic materials ${ }^{19-20}$, they quickly enter the food chain as contaminants reaching humans through the consumption of drinking water and agricultural food products ${ }^{8-11}$. Although most of them have been

*Corresponding author: Ahmed Lakhili

Email address : ahmedlakhili@gmail.com DOI: http://dx.doi.org/10.13171/mjc851907031al banned from use, they are still detected in ecosystems ${ }^{9-10}$.

Recently, the analysis of pesticide residues has received increasing attention in different parts of the world, including in North African countries. In particular, it has been remarked that the pesticides ${ }^{16-22}$, monitoring in industrial products is very important and requires high precision, unique selectivity and high sensitivity techniques ${ }^{12-13}$.

In agribusiness industries, the use of pesticides (rat poisons and insecticides) in the treatment of production and storage areas eliminates the apparition of pests that may impair the quality of the finished products or the production process. The law frames the latter and controlled by the ONSSA (National Office of Sanitary Safety of Food Products) within the framework of effective normative use of such chemical products, without an impact on industrial food products ready for consumption.

In this present work, we first verify the impact of the use of these products (rat poisons, insecticides) used to treat pests in closed industrial areas (storage areas, production areas). Then, we check the

Received April 27, 2019 Accepted June 10, 2019 Published July 3, 2019 
presence or the absence of traces and concentrations of these products in the finished products. For this purpose, we adopt the method of the microextraction on the solid phase SPME, with the use of the chromatography gas coupled to mass spectroscopy (GC-MS). This coupling SPME/GCMS provides excellent sensitivity and precision even at deficient concentrations.

\section{Materials and methods}

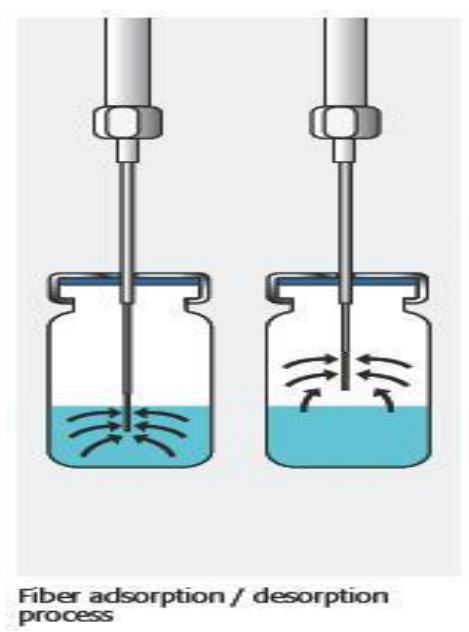

In this section, we give the essential on the used methods, including materials.

\section{SPME solid phase micro-extraction method}

For the detection and the quantification of molecules of the industrial products by the solid phase microextraction method (SPME), we immersed the SPME fiber in the studied samples. This step was carried out at room temperature for 15 minutes. The procedure is illustrated in (Figure 1).

Figure 1. Adsorption and desorption process by SPME

The used fiber consists of a Carboxen/PDMS/DVB 50/30 $\mu \mathrm{m}$ phase. The chemical compounds are first concentrated on the fiber (adsorption phase), then they are thermally desorbed in the GC-ECD injector. The analysis conditions are the same as the ones mentioned above $21-22$.

\section{Optimizing SPME}

To increase the sensitivity and the effectiveness of the SPME method, optimization of various parameters such as the temperature, the exposure time, the duration and the mode of agitation, the effect of the $\mathrm{pH}$ and adding salt was performed.

\section{Calculation method}

The concentration is determined according to the method of qualification of peaks, taking into account the volume of the final extract and the volume of the sample analyzed for each identified molecule. For the SPME

the method, the concentration is calculated using the following equation:

$\mathrm{Ce}=\frac{\mathrm{Ae} \times \mathrm{Ci}}{\text { Ast }}$

Where Ce is the concentration of a compound in the sample. Ae indicates the air of a compound in the sample. Ast is the peak air of the studied compound. $\mathrm{Ci}$ represents the initial concentration of the studied

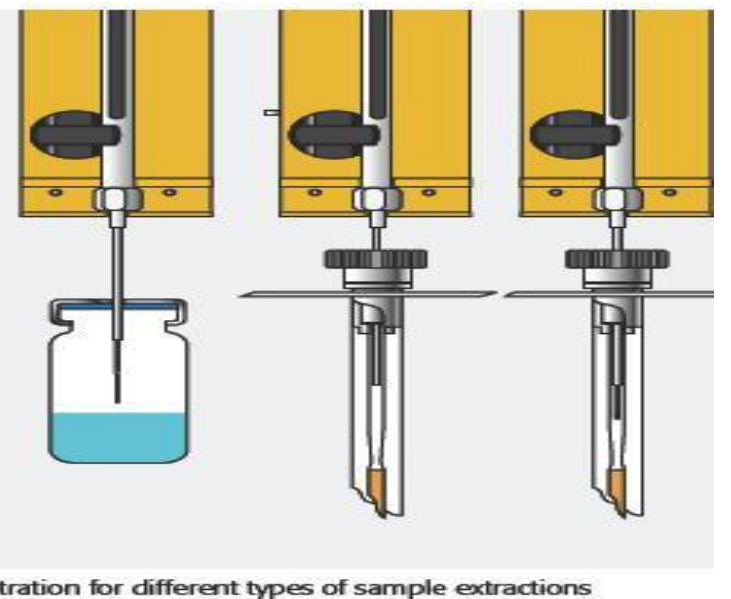

compound and $\mathrm{V}$ denotes the volume of the sample to be dosed in $\mathrm{mL}$. Ve is the volume of extract in $\mathrm{mL}$. The percent recovery (PR\%) or yield of each compound was calculated using the peak air. Concretely, this has been done using the following equation:

$\mathrm{PR}=\frac{\mathrm{Ce}}{\mathrm{Ci}} \times 100$

Where Ce represents the concentration of the sample and $\mathrm{Ci}$ denotes the standard initial concentration.

\section{Results and Discussions}

In this section, we present the obtained results and the corresponding discussions.

The stability and validity of the used method were checked before generating any results in this investigation.

The validation of an analytical method consists of the determination relevant parameters as the fidelity, which itself represents a set of dispersion characteristics including repeatability, intermediate fidelity and reproducibility.

As criteria for estimating the stability, an examination repeatability test was performed. It consists of testing to evaluate stability; a repeatability test was performed. It consists of testing the same size in conditions as stable as possible and 
at close intervals. This measure of the variation of the results is carried out under the same conditions. The precision can be obtained when the same investigator repeats the method under the same conditions (reagents, equipment, adjustment and laboratory) in a short time interval. This makes it possible to evaluate the accuracy of the method under normal operating conditions.

To study the SPME stability, a series of five repetitions analysis was performed on the chemical compost concentration $(5 \mu \mathrm{g} / \mathrm{L})$ under optimized conditions. The obtained results are shown (Figure 2).

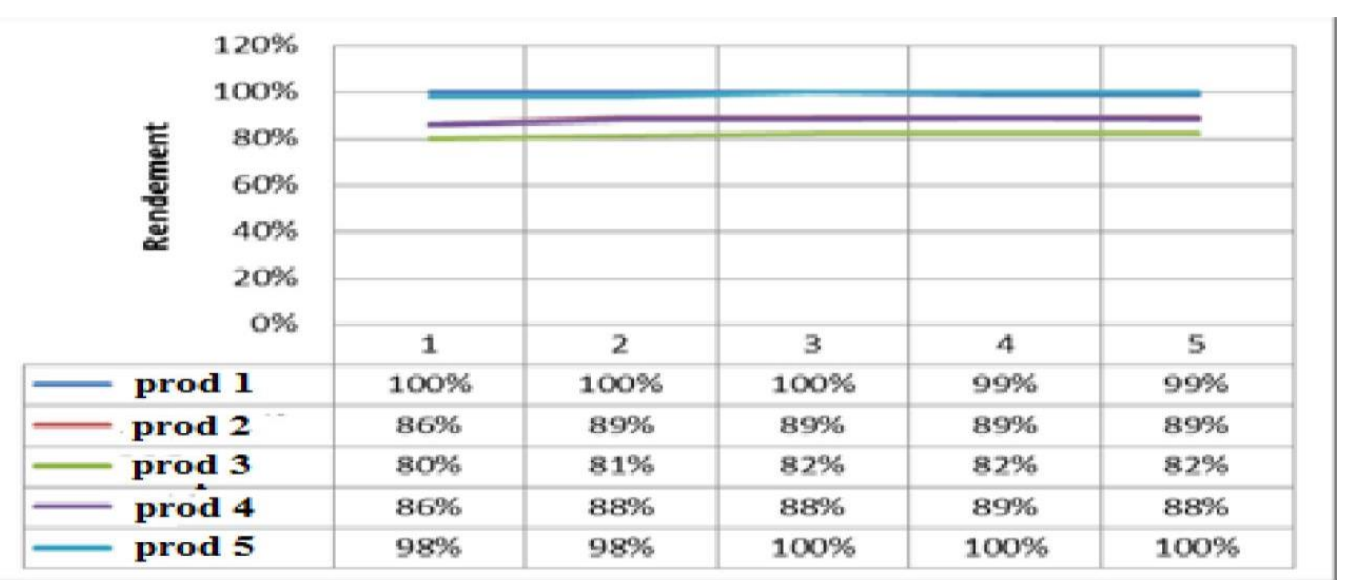

Figure 2. Variation stability of SPME a series of five repetitions analysis was performed on the chemical compost concentration $(5 \mu \mathrm{g} / \mathrm{L})$

From Figure 2, it can be seen that for each product, there is no variation with an identical yield value obtained for all tests.

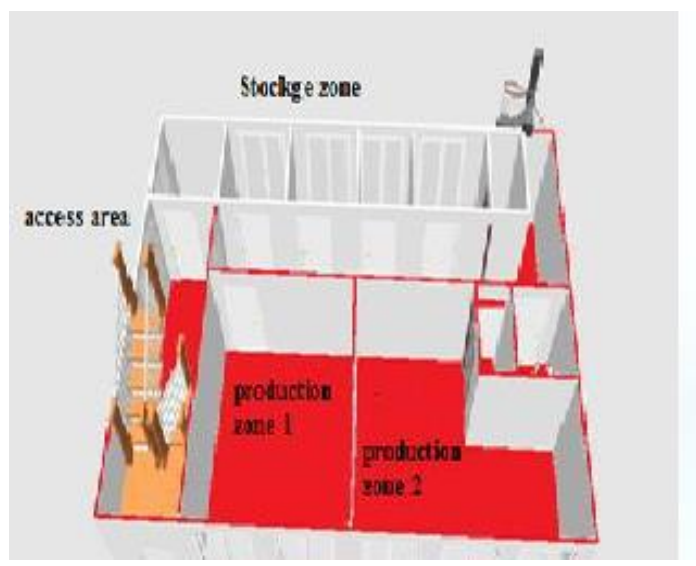

With the method, stability checked, the samples were analyzed. The studied industrial areas are given in Figure 3.

Figure 3. The overall scheme of the building of products and storage treated by chemicals

Having discussed the stability of five products, we present now the results and give the corresponding discussions.

Production areas (ZONE 1 and ZONE 2) were treated by spraying a liquid insecticide at the end of the production at $9 \mathrm{pm}$. The treated products are illustrated in Figure 4.

Several varieties of insecticides were used in this study. First, a profiling of the active ingredient of each industrial formulation was performed (rat poison/insecticide). To be able to identify them, an analysis study should be performed. Indeed, samples are taken randomly in all the products before and after the surface treatment to evaluate the impact of the treatment in the quality of the finished products.

After treatment, the SPME fiber was implanted at the oven outlet of the cooking belt, to take advantage of the optimal adsorption conditions already established in our preliminary technique implementation study.

The following results represent the analytical profiles of the different peaks found by SPME/GC analysis: MS (Figure 5). 


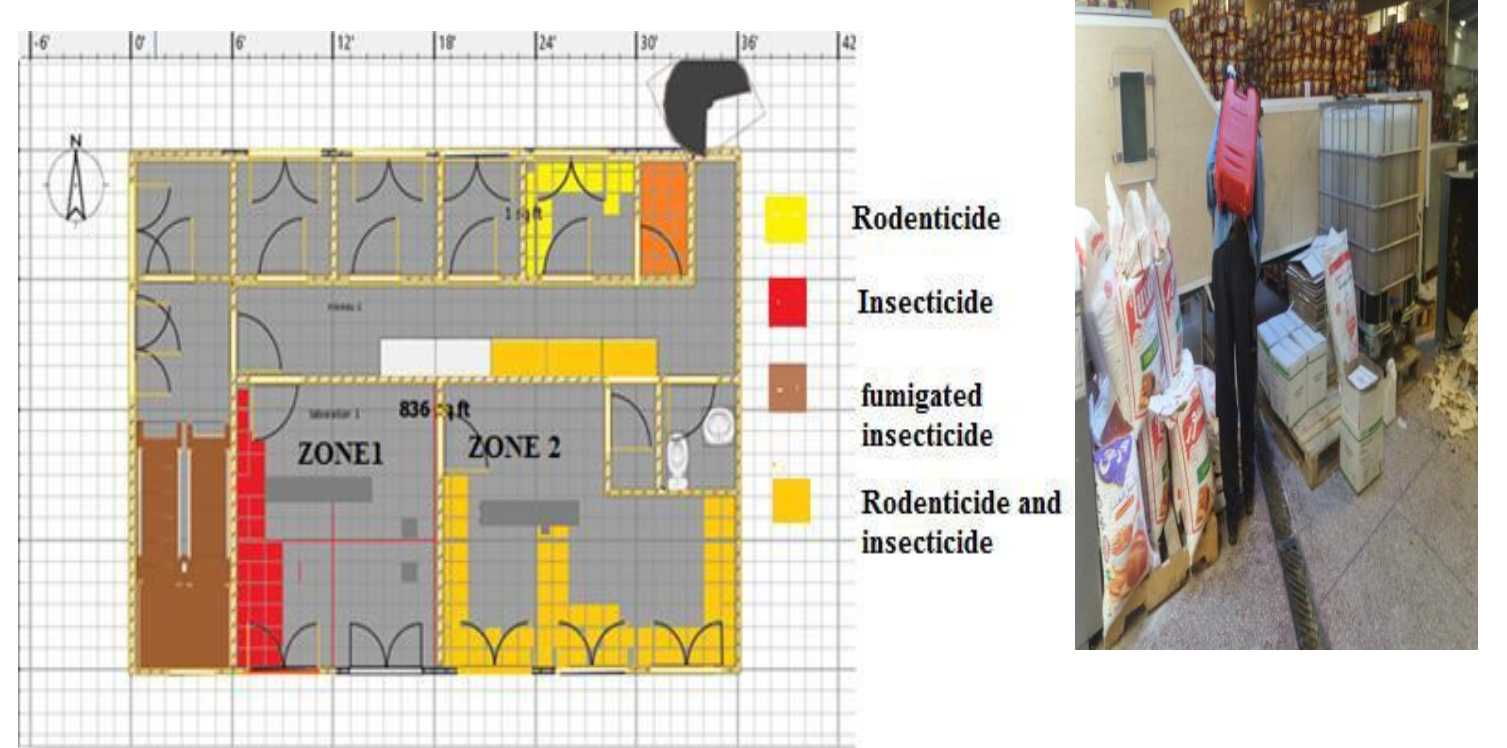

Figure 4. Distribution of treatment areas by type of product used

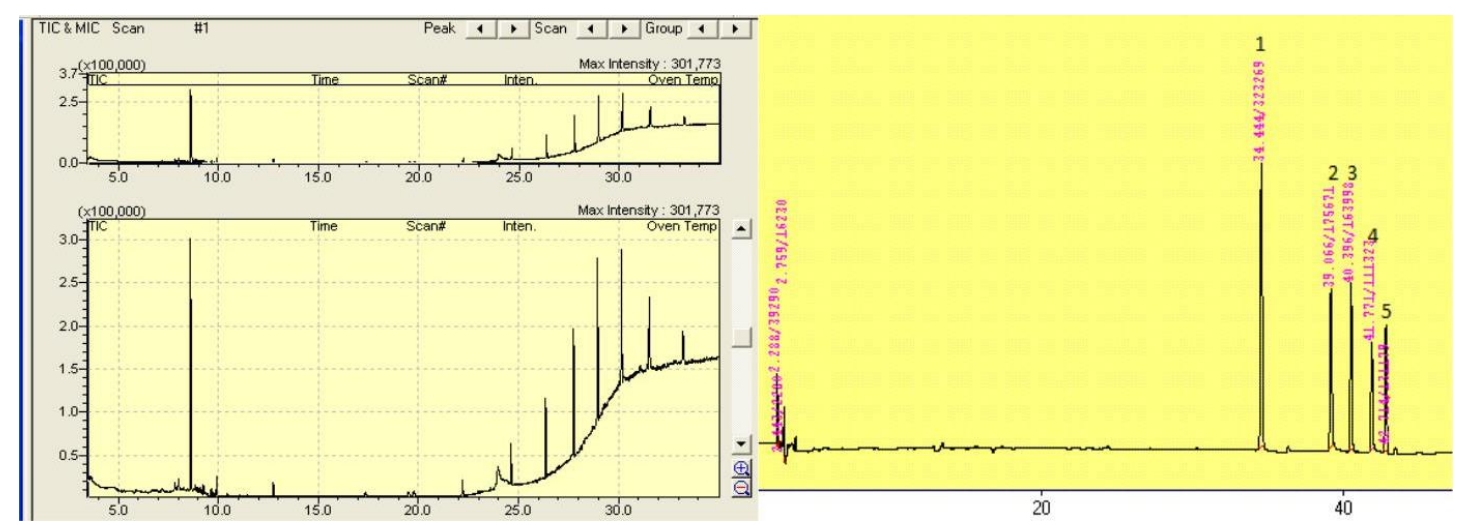

Figure 5. Profile of the treatment product obtained by SPME coupled with GC-MS and GC-ECD

Figure 5 represents the profile of the different chemical molecules that are present in the industrial formulation for insect treatment, analyzed by SPME coupled to a GC-ECD and GC-MS.
In what follows, we discuss the product analysis after 6 hours spent from the moment of surface treatment with insecticide raticide. Figure 6 represents the results obtained after the analysis of the food products produced in zone 1 after 6 hours of the surface treatment. (Figure 6).

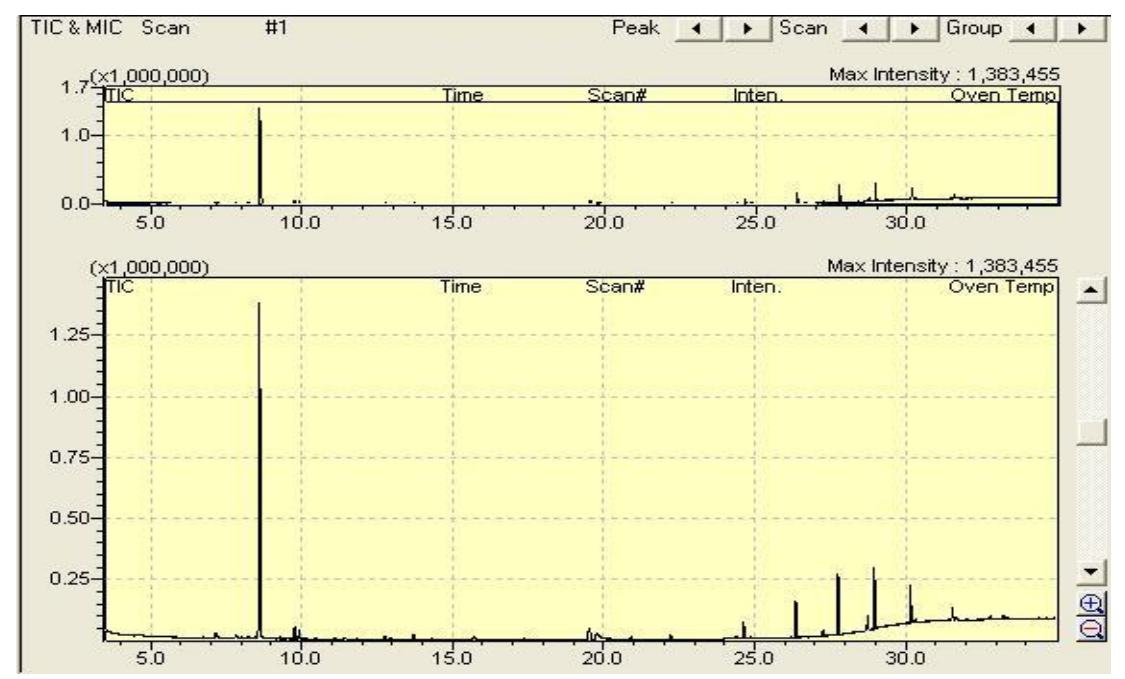

Figure 6. Analysis results of the finished product by SPME coupled to GC-MS after 6 hours of treatment 
After 6 hours of the surface treatment by spraying using pesticides and rat poisons, the presence of certain chemical products at low concentration was observed. This implies that the 6 hours' treatment is not sufficient to eliminate these chemicals by spray.
Figure 7 shows the results obtained by SPME coupled to GC-MS after analysis of industrial production in a firm surface treated by spraying for 12 hours.

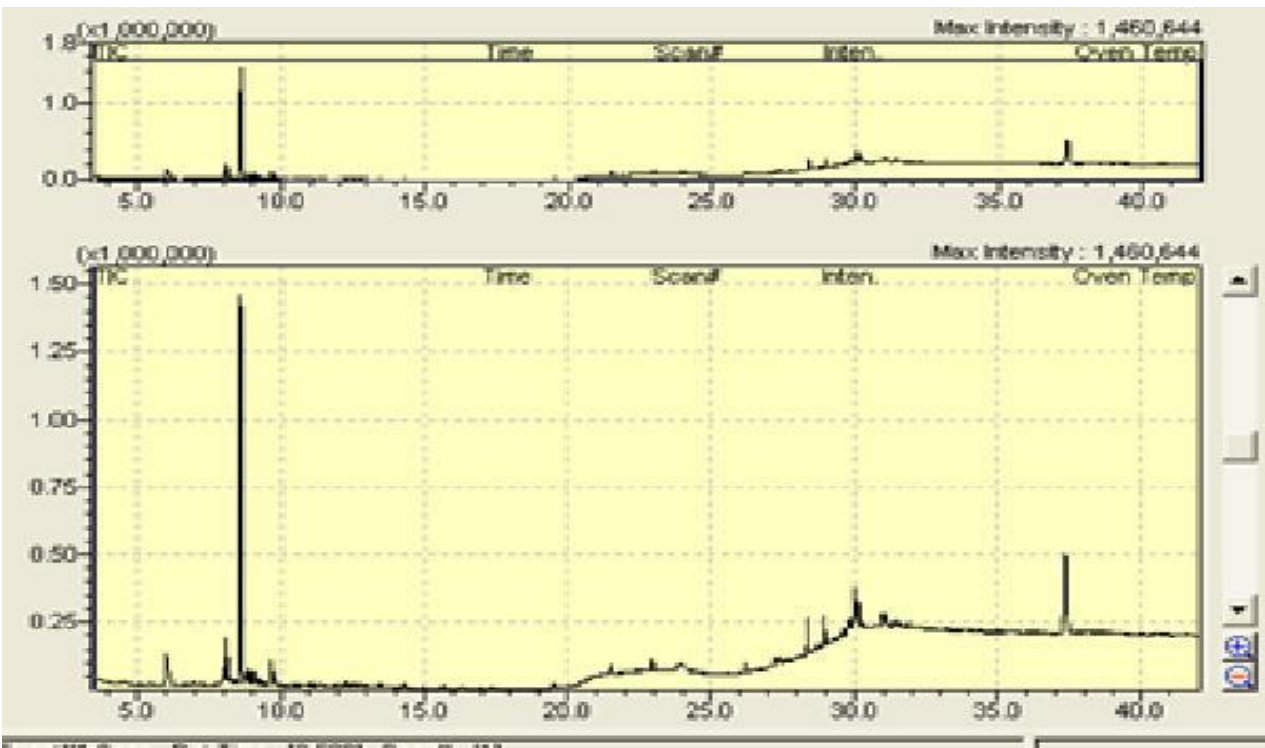

Figure 7. Analysis results of the finished product by SPME coupled to GC-MS after 12 hours of treatment

After 12 hours of surface treatment, two molecules were present at low concentrations. It has been shown that the resumption of production after 12 hours of surface treatment can present risks on the quality of the finished product. The results obtained by SPME coupled to GC-MS of firm surface treatment for 18 hours are displayed in Figure 8.

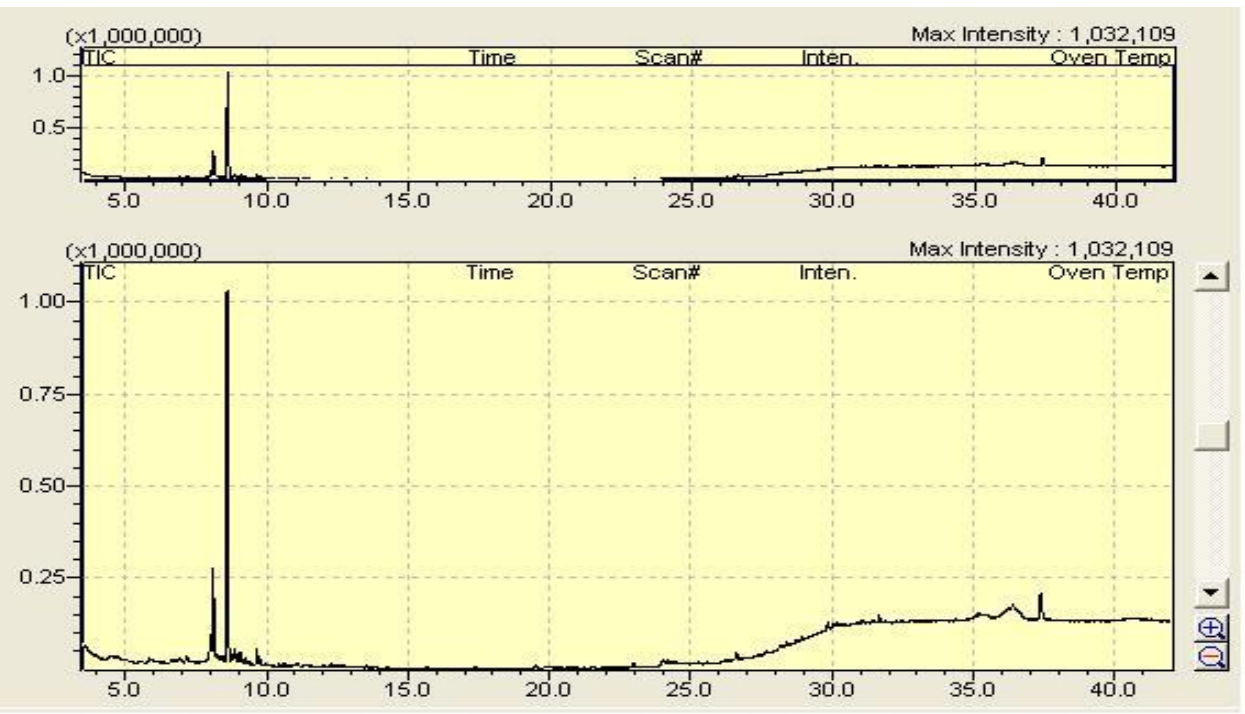

Figure 8. Analysis results of the finished product by SPME coupled to GC-MS after 18 hours of treatment

It is noted that after 18 hours of surface treatment, the presence of surface treatment products in the finished industrial products can be neglected. These observations suggest that an $18 \mathrm{~h}$ treatment time is a minimum period needed to eradicate most of the undesirable chemicals left in the product.
The results obtained by SPME coupled to GCMS after analysis of industrial production in a firm surface after 24 hours of surface treatment by spraying are illustrated in Figure 9. 


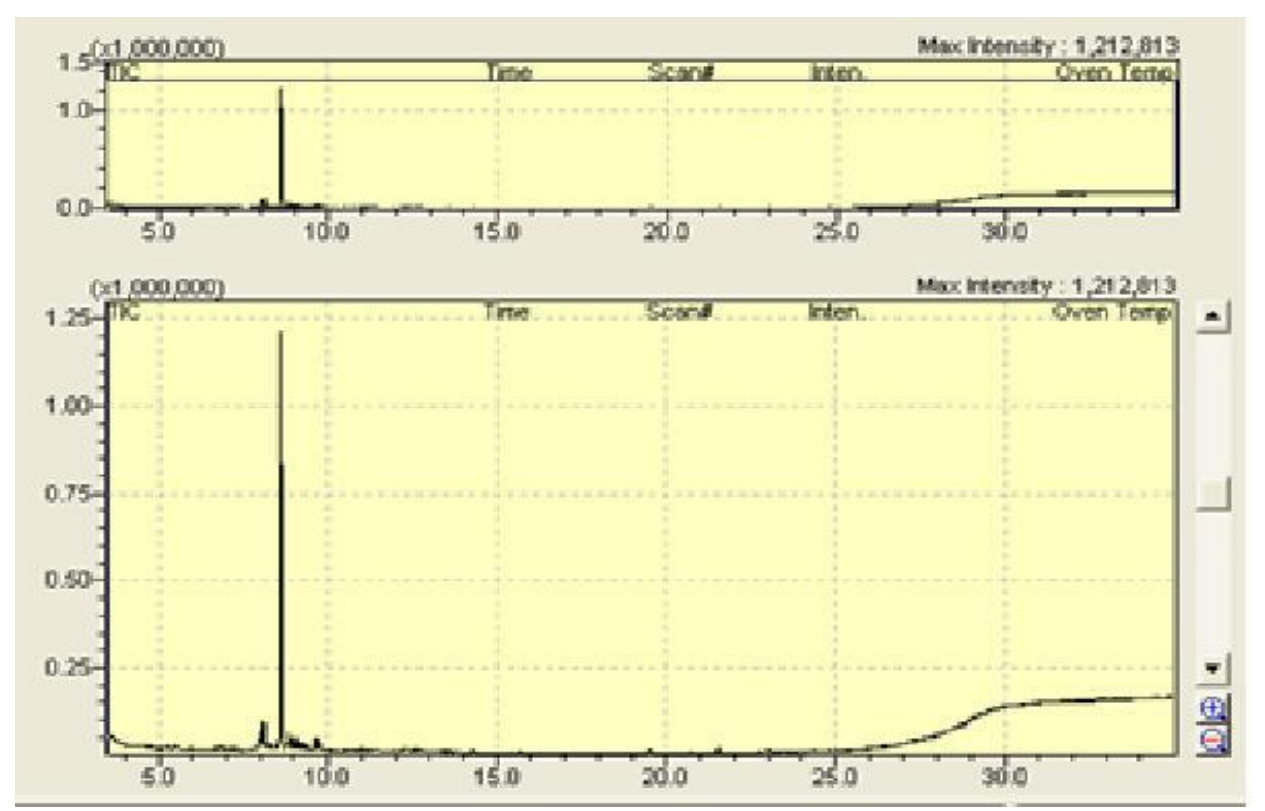

Figure 9. Analysis results of the finished product by SPME coupled to GC-MS after 24 hours of treatment

After 24 hours of surface treatment, a complete absence of undesirable chemicals is observed.

Several pest control products used for closed production surfaces were studied, along with their impact on the quality of production. Several industrial formulation profiles of insecticides and rat poisons were developed using the SPME technique coupled to GC-MS with repeatability and reproducibility, in order to establish and verify the validity of the analytical method adopted. The present study respects the ISO quality norms. It has been observed that the use of these products may hurt the quality of the agri-food production treated by chemical spraying in the first 6 hours after treatment.

It has also been observed that the use of SPME coupled to GC-MS in the routine quality analysis is an economical, efficient and stable method concerning repeatability and reproducibility. It allows for accurate identification of all elements present at different concentrations, even at traces levels.

\section{Conclusion}

The present work was focused on the study of the impact of rat poison and insecticides in closed industrial facilities (storage areas, production areas). The main focus was to use micro-extraction on the solid phase SPME in the presence of the chromatography gas coupled to a mass spectrum (GC-MS) to reveal the presence of chemicals traces.

Several assays were carried out by SPME coupled to the GC-MS on a sample product in a closed environment, treated with rat poison, taken at different times with an interval of 6 hours, according to the iso quality standard adopted by the agri-food production. It has been shown that the interval of $18 \mathrm{~h}$ of production break after chemical treatment is necessary to avoid the presence of remaining traces of the said chemical. Thus, avoiding the alteration of the finished product. It was also revealed that the coupling SPME/GC-MS provides great sensitivity and precision even at low concentrations.

\section{References}

1- A. Lakhili, M. Fekhaoui, A. Bellaouchou, A. El Abidi, L. Tahri, SPME Extraction Methods of Doped Organochlorine Pesticides in Moroccan Water Samples, Mediterr. J.Chem., 2018, 7(4), 294-302.

2- R.I. Bonansea, M.V. Amé, D.A. Wunderlin, Solid-phase microextraction and gas chromatography with mass spectrometric detection for the determination of pesticides in aqueous samples, Journal of Chromatography A, 2013, 795, 105-115.

3- C. L. Arthur, J. Pawliszyn, Solid Phase Microextraction with thermal desorption using fused silica optical fibers, Analytical Chemistry, 1990, 62, 2145-2148.

4- R. A. Dong, P. L. Liao, Determination of organochlorine pesticides and their metabolites in soil samples using headspace solid-phase microextraction, Journal of Chromatography A, 2001, 918, 177-188.

5- C. P. Dougherty, S. H. Holtz, J. C. Reinert, L. Panyacosit, D. A. Axelrad, T. J. Woodruff, Dietary exposures to food contaminants across the United States, Environmental Research, 2000, 84, 170-180.

6- J. H. Jelen, M. Majcher, M. Dziadas, Multiresidue detection of pesticides in juice and fruit 
wine: A review of extraction and detection methods, Food Research International, 2012, 46, 399-409.

7- R. P. Berladi, J. Pawliszyn, The application of chemically modified fused silica fibers in the extraction of organics from water matrix samples and their rapid transfer to capillary columns Environmental Science and Pollution Research, 1989, 24, 179-191.

8- R. Botter, G. Bouchoux, Techniques de l'ingénieur, Spectrométrie de masse, Determination of chlorinated pesticides in water by SPME/GC, Water Research, 2002, 36, 1909-1911.

9- H. Shahsavand, M. R. Nateghi, Analysis of chlorothalonil and degradation products in soil and water by GC/MS and LC/MS. Chemosphere, Journal of Water Chemistry and Technology, 2018, 04, 86-90.

10-S. Chen, S. Lili, S. Zhengjun, H. Qiuhui, Determination of organochlorine pesticide residues in rice and human and fish fat by simplified two-dimensional gas chromatography, Food Chemistry, 2007, 104, 1315-1319.

11-C. De Rossi, R. Bierl, J Riefstahl, Organic pollutants in precipitation: monitoring of pesticides and polycyclic aromatic hydrocarbons in the region of Trier (Germany), Physics and Chemistry of the Earth, 2003, 28, 307-314.

12-A. Derouiche, M. R. Driss, J. P. Morizur, M.H. Taphanel, Simultaneous analysis of polychlorinated biphenyls and organochlorine pesticides in water by headspace solid-phase microextraction with gas chromatographytandem mass spectrometry, Journal of Chromatography A, 2007, 1138, 231-300.

13-M. Fernández, C. Padrón, L. Marcon, S. Ghini, R. Colombo, A. G. Sabatini, S. Girotti, Determination of organophosphorus pesticides in honeybees after solid-phase microextraction, Journal of Chromatography A, 2001, 922, 257-265.

14-C. Ferrer, M. J. Gómez, J. F. García-Reyes, I. Ferrer, E. M. Thurman, A.R. Fernández-Alba, Determination of pesticide residues in olives and olive oil by matrix solid-phase dispersion followed by gas chromatography/mass spectrometry and liquid chromatography/tandem mass spectrometry, Journal of Chromatography A, 2005, 1069, 183-194.
15-J. Fillion, F. Sauvé, J. Selwyn, Multiresidue method for the determination of residues of 251 pesticides in fruits and vegetables by gas chromatography/mass spectrometry and liquid chromatography with fluorescence detection, Journal of AOAC INTERNATIONAL, 2000, 83, 698-750.

16-H. Shahsavand, M. R. Nateghi, Solid Phase Extraction and Flame Atomic Absorption Determination of Cadmium in Water Samples, Journal of Water Chemistry and Technology, 2018, 86-90.

17-S.A. Harris, M.J. Whiticar, M.K. Eek, Molecular and isotopic analysis of oils by solid phase microextraction of gasoline range hydrocarbons, Organic Geochemistry 1999, 30, 721-737.

18-F. Hernández, O. J. Pozo, J. V. Sancho, L. Bijlsma, M. Barreda, E. Pitarch, Multiresidue liquid chromatography-tandem mass spectrometry determination of 52 non-gas chromatography-amenable pesticides and metabolites in different food commodities, Journal of Chromatography A, 2006, 1109, 242-252.

19-M.C. Sampedro, O. Martin, C. Lopez de Armentia, M.A. Goicolea, E. Rodriguez, Z. Gomez de Balugera, J. Costa-Moreira, R. J. Barrio, Solid-phase microextraction for the determination of systemic and non-volatiles pesticides in river water using gas chromatography with nitrogen-phosphorus and electron-capture detection, Journal of Chromatography A, 2000, 893, 347-358.

20-F. J. Santos, M. T. Galceran, The application of gas chromatography to environmental analysis Trends in Analytical Chemistry. 2002, 2, 672-685.

21- A. Yang, J. Zhao, J. Wang, H. Yu, H. Piao, D. Li, Water-based gas purge microsyringe extraction coupled with liquid chromatography for determination of alkylphenols from seafood Laminaria japonica Aresh, Journal of Chromatography A, 2018,1300, 302-360.

22-J.G. Eskenazi, E. A. Gladstone, A. Bradman, L. Pedersen, C. Johnson, D. B. Barr, Scientific Reports volume 8., Association between in utero organophosphate pesticide exposure and abnormal reflexes in neonates, NeuroToxicology, 2018, 26, 1167-1180. 